\title{
Segregación escolar por nivel socioeconómico en Colombia y sus departamentos
}

School Segregation by Socioeconomic Level in Colombia and its Departments

Artículo de investigación | Research article

Fecha de recepción: 12 de agosto de 2019 Fecha de aceptación: 30 de octubre de 2020 Fecha de disponibilidad en línea: julio de 2021

doi: 10.11144/Javeriana.m14.sens

F. JaVier MuriLlo javier.murillo@uam.es

Universidad Autónoma de Madrid, España D ORCID: https://orcid.org/0000-0002-8003-4133

SAndRa CARrillo-Luna

carrillo.sandra@pucp.pe

Pontificia Universidad Católica del Perú, Perú iD ORCID: https://orcid.org/0000-0003-2871-4702

Para citar este artículo | To cite this article Murillo, F. J. \& Carrillo-Luna, S. (2021). Segregación escolar por nivel socioeconómico en Colombia y sus departamentos. magis, Revista Internacional de Investigación en Educación, 14, 1-23. doi: 10.11144/Javeriana.m14.sens 


\title{
Resumen
}

El objetivo de este estudio es estimar la magnitud de la segregación escolar en Colombia y sus departamentos, tanto en educación primaria como en secundaria inferior. Para ello, se hace una explotación especial de los datos de las pruebas censales Saber de 2016 para estudiantes de $5^{\circ}$ y $9^{\circ}$ grado. La muestra está conformada por 1138885 estudiantes, matriculados en 24401 escuelas. Los resultados indican que la magnitud de la segregación escolar es alta, tanto para primaria como para secundaria, con tendencia a una mayor segregación en los grupos de más nivel socioeconómico, así como diferencias significativas entre los departamentos.

\section{Palabras clave}

Colombia; educación básica; estatus socioeconómico; igualdad de oportunidades educativas; segregación escolar

\begin{abstract}
This paper aimed to estimate the magnitude of school segregation in Colombia and its departments, both in primary and lower secondary education. An analysis of the 2016 Saber census tests for 5 th and 9 th grade students was made. The sample was made up of 1,138,885 students, enrolled in 24,401 schools. Results indicated that the magnitude of school segregation was high, both for primary and secondary levels, with a trend towards greater segregation in groups with a higher socioeconomic level, as well as significant differences between departments.
\end{abstract}

\section{Keywords}

Colombia; basic education; socio-economic status; universal education; school segregation 
Descripción del artículo | Article description

Artículo de investigación derivado del proyecto Escuelas

en contextos socioeconómicamente desafiantes: una

aproximación desde la educación para la justicia social.

\section{Introducción}

Los datos disponibles indican que, en estos últimos años, la segrega-

Tener un sistema educativo con una alta segregación escolar trae devastadoras consecuencias para los estudiantes y para la sociedad. Todas las evidencias disponibles apuntan a que la segregación afecta el aprendizaje académico de los estudiantes menos favorecidos (p. e., Agirdag et al., 2013; Dupriez \& Dumay, 2006; Gorard, 2014; Granvik-Saminathen et al., 2018; Palardy, 2013), pero también su desarrollo integral y su socialización. Igualmente, un sistema educativo segregado solo contribuirá a una sociedad excluyente e inequitativa (Mickelson, 2018; Valenzuela et al., 2014). Así, si la evidencia internacional demuestra que la segregación no solamente reduce el desempeño académico de los estudiantes, sino que, además, afecta negativamente la cohesión social (Organisation for Economic Co-operation and Development, 2012), las inequidades del sistema educativo colombiano podrían ser causa y consecuencia de esa segregación.

Sin embargo, los pocos estudios recientes que han analizado la magnitud de la segregación escolar en Colombia proceden de evaluaciones internacionales como la de PISA (educación secundaria inferior) o el Tercer 
Estudio Regional Comparativo y Explicativo (Terce) (educación primaria), que al utilizar muestras dan una imagen muy general, con pocos matices y con poca precisión. En esta investigación, se pretende estimar la magnitud de la segregación escolar en Colombia, tanto en educación primaria ( $5^{\circ}$ grado) como secundaria inferior ( $9^{\circ}$ grado), usando las pruebas censales de Saber del 2016, del Instituto Colombiano para la Evaluación de la Educación (Icfes).

\section{Antecedentes}

Existe un progresivo consenso en entender por segregación escolar la distribución desigual de los estudiantes en las escuelas en función de sus características personales, sociales o de sus condiciones. Esta definición hace referencia a la llamada dimensión de uniformidad de la segregación escolar, que es el enfoque utilizado por la gran mayoría de los trabajos que estiman su magnitud en América Latina (Krüger, 2019; Murillo, 2016; Murillo \& Martínez-Garrido, 2017; Murillo et al., 2018; Vazquez, 2016). Para su estimación, se cuenta con una miríada de índices: desde el clásico de disimilitud (o de Duncan), pasando por el índice de raíz cuadrada (o de Hutchens), el índice de información mutua, el índice de brecha por centiles (CGI) o el más reciente índice de Gorard. Aunque cada uno de estos índices tiene sus particularidades, ventajas y limitaciones, la imagen que todos ellos nos ofrecen de la segregación es análoga (véase Murillo, 2016).

Una concepción alternativa de segregación escolar se obtiene de la consideración de su dimensión de exposición. Con ella, se entiende la segregación como la probabilidad de que un estudiante de un grupo minoritario se encuentre en una escuela con alguien de su mismo colectivo. Su uso ha sido mucho menos habitual en América Latina y siempre como complemento al estudio de la dimensión de uniformidad (p. e., Krüger, 2019; Murillo, 2016; Vazquez, 2016). En este caso se usa siempre el índice de aislamiento.

Bien sea desde la dimensión de uniformidad o desde la dimensión de exposición, es posible distinguir diversos tipos de segregación escolar según sea el criterio utilizado. Es decir, según sean las características personales o sociales o las condiciones de los estudiantes que generan esa distribución desigual, o del criterio de conformación del grupo minoritario. Así, los primeros estudios relacionados, realizados a finales de los años 50 en Estados Unidos, abordaban la segregación escolar como la pertenencia a un grupo etnorracial; posteriormente, en Europa se desarrollaron trabajos que buscaban estimar la magnitud según el origen nacional de los estudiantes, y, más recientemente, ya en todo el mundo, se inició la preocupación por la 
segregación por origen socioeconómico de las familias de los estudiantes. También es posible analizar la segregación en función del rendimiento previo de los estudiantes o de las necesidades educativas especiales o incluso de género, pero este tipo de trabajos apenas se ha desarrollado.

La investigación sobre segregación escolar en América Latina, especialmente aquella que busca estimar su magnitud, es muy reciente, menos de 20 años. Esta se ha desarrollado esencialmente con la disponibilidad de bases de datos generadas por evaluaciones internacionales, como PISA o Terce; o nacionales, como el Sistema de Medición de la Calidad de la Educación (Simce), en Chile; la Evaluación Censal de Estudiantes (ECE), en Perú; Ser Estudiante y Ser Bachiller, del Instituto Nacional de Evaluación Educativa (Ineval), en Ecuador; Aristas, de Uruguay; la Evaluación Diagnóstica Nacional, de República Dominicana, o Saber, del Icfes, en Colombia, por poner algunos ejemplos.

Los estudios que estiman la magnitud de la segregación escolar en Colombia son escasos y recientes, y siempre han sido explotaciones especiales de las bases de datos del PISA o del Terce.

Así, por señalar algunos, se destaca, en primer lugar, el trabajo de Duarte et al. (2012). Estos investigadores realizan un estudio sobre la equidad en la educación colombiana a partir de una explotación de la base de datos Saber de 2009. En ella, aunque no estiman la segregación tal y como se ha venido señalando en este trabajo, aportan resultados interesantes y afirman que "los hallazgos presentados indican que en Colombia existe un alto grado de segregación de las escuelas según el NSE [nivel socioeconómico]" (p. 17), a lo que añaden que "los resultados de los estudiantes en las pruebas están altamente relacionados con dicha segregación". En su texto no aportan mucha más información, pero es un buen punto de partida que alerta sobre la situación de segregación y destaca la importancia de este hecho para el aprendizaje de los estudiantes. Mucho más recientemente, se cuenta con explotaciones del Terce, para educación primaria, y del PISA 2015, para la secundaria.

En educación primaria, F. Javier Murillo (2016), con base en los datos del Terce, encuentra que Colombia tiene una segregación escolar promedio alta: de 0,59, según el índice de disimilitud; de 0,40, según el índice de Gorard y de 0,54, según el índice de aislamiento. Estas cifras se ven corroboradas en el posterior estudio de Murillo \& Martínez-Garrido (2017), quienes estiman la segregación escolar por nivel socioeconómico con los datos del Terce, mediante el índice de disimilitud. Ellos encuentran que para el 25\% de los estudiantes con familias de menor nivel socioeconómico y cultural (Q1), el índice promedio de disimilitud es de 0,53 y para el $25 \%$ de mayor nivel, el 0,66, siendo este último el más alto de América Latina y el Caribe. 
Para la educación secundaria, Emmanuel Vazquez (2016) utiliza los datos del PISA del año 2015 para cuantificar los niveles y la evolución de la segregación escolar por nivel socioeconómico de los 72 países que participaron en la evaluación internacional, a través de cuatro índices: disimilitud, de aislamiento, de raíz cuadrada y el índice de brecha por centiles. Entre sus resultados, encuentra que seis países de América Latina (entre ellos Colombia) se encuentran entre los diez más segregados, entre los estudiados. Concretamente, estima que Colombia tiene una segregación escolar de 0,44, usando el índice de disimilitud; de 0,65, usando el índice de aislamiento; de 0,21, usando el de raíz cuadrada y de 0,32, usando el índice de brecha por centiles; sin embargo, para estimar los tres primeros no informa cómo eligió el grupo minoritario.

Murillo et al. (2018), por su parte, hacen un estudio de la evolución de la segregación escolar en América Latina, utilizando también el PISA 2015, y encuentran que la segregación escolar en Colombia ha tenido una tendencia al alza en los últimos años, siendo uno de los países de América Latina en los que más ha aumentado. En el año 2015, la segregación estimada mediante el índice de Gorard fue de 0,26, para el P10; de 0,43, para el Q1; de 0,51, para el Q4 y de 0,59, para el P90.

Por último, la investigadora argentina Natalia Krüger (2019), de nuevo con datos del PISA 2015, encuentra valores promedio de segregación para Colombia entre 0,46 y 0,51, con el índice de disimilitud; entre 0,35 y 0,53, con el índice de aislamiento y de 0,28, con el índice de información mutua. Esta investigadora confirma que en Perú, Chile y Colombia los niveles de segregación han crecido a lo largo de los quince años analizados (2000-2015), con aumentos trianuales promedio entre un 3 y un $8 \%$, aproximadamente, siendo estos tres países, junto con México, los que encabezan los rankings regionales por su mayor segregación.

La disparidad encontrada tiene su justificación en la dispersión en la forma de medir la segregación, no solo en la dimensión de la segregación antes señalada, sino también en el índice utilizado (p. e., el índice de Gorard da resultados más bajos que el de disimilitud) o en el grupo minoritario considerado (P10 y Q1 para los grupos de menores nivel socioeconómico, Q4 y P90 para los de mayor nivel). De esta forma, las estimaciones de la segregación cobran mayor sentido si son interpretadas en comparación con otras realidades, países o contextos, más que en su forma absoluta. Sea como fuere, los datos de la magnitud de la segregación escolar en Colombia son siempre muy generales y han sido obtenidos siempre a partir de datos muestrales.

Colombia, según datos del Banco Mundial de 2017, es el segundo país más inequitativo de América Latina, y el séptimo de todo el mundo, siendo 
su índice de Gini de 0,517 en 2018. Esta desigualdad se refleja en una gran brecha entre departamentos urbanos y rurales, siendo estos últimos en donde se concentra una gran parte de la pobreza del país y también la mayor desigualdad (Departamento Administrativo Nacional de Estadística, 2016). El sistema educativo colombiano atiende a 10036440 estudiantes en la educación formal, de los cuales un $80,4 \%$ son del sector público y un $19,6 \%$, del privado, con una gran diferencia por grados y departamentos. Así, en básica primaria, con 3617484 estudiantes, el 76,9\% estudian en centros públicos; sin embargo, para el departamento de Chocó esta cifra es del 97,7\% y para el distrito capital de Bogotá es del 60,9\%. Para la básica secundaria, los datos son análogos, de los 2976034 estudiantes matriculados en el conjunto del país el $81,8 \%$ estudia en centros públicos. Sin embargo, en Chocó este porcentaje es del 98,3\% y en el distrito capital de Bogotá es del 63,6\% (Departamento Administrativo Nacional de Estadística, 2019).

En esta investigación se pretende aportar una imagen completa e inédita de la magnitud de la segregación escolar en Colombia a partir de tres estrategias complementarias:

1. Estimar la magnitud de la segregación escolar por nivel socioeconómico en educación primaria y secundaria en Colombia a través de los cuatro índices más habituales (disimilitud, Gorard, raíz cuadrada y aislamiento).

2. Determinar los perfiles de segregación escolar por nivel socioeconómico en Colombia para primaria y secundaria.

3. Cuantificar la segregación escolar de los 32 departamentos, más el distrito capital de Bogotá, en los que está dividido Colombia.

\section{Metodología}

Con el fin de dar respuesta al objetivo formulado en esta investigación, se realiza una explotación especial de la base de datos de la prueba Saber, desarrollada por el Icfes, correspondiente al año 2016.

La prueba Saber es una evaluación del aprendizaje de los estudiantes de $3^{\circ}, 5^{\circ}$ y $9^{\circ}$ grado que desarrolla el Icfes anualmente. Esta prueba es de carácter censal, por lo que se aplica a todos los estudiantes de esos grados. En esta investigación solo se utilizaron datos de $5^{\circ}$ grado (educación primaria) y de $9^{\circ}$ grado (educación secundaria inferior). Junto con las correspondientes pruebas de aprendizaje de lenguaje, matemáticas, ciencias naturales y competencias ciudadanas, se aplica una prueba de factores asociados, lo que permitió la realización de este estudio. 
La muestra total fue de 1138885 estudiantes analizados, que estudian en 24401 escuelas, repartidas en las 33 regiones educativas en las que está dividida Colombia (32 departamentos y el distrito capital de Bogotá). De ellos, 646413 son estudiantes de $5^{\circ}$ grado, distribuidos en 14630 escuelas, y los restantes 492472 son estudiantes de $9^{\circ}$ grado, distribuidos en 9771 escuelas. En la tabla 1 se detalla el número de estudiantes y de escuelas en cada departamento, así como el promedio del índice socioeconómico (ISE) de las familias de los estudiantes, tanto en $5^{\circ}$ como en $9^{\circ}$ grado.

Tabla 1

Muestra y sus características

\begin{tabular}{|c|c|c|c|c|c|c|}
\hline & \multicolumn{3}{|c|}{$\begin{array}{l}\text { Educación primaria } \\
\text { (5º grado) }\end{array}$} & \multicolumn{3}{|c|}{$\begin{array}{l}\text { Educación secundaria inferior } \\
\text { ( } 9^{\circ} \text { grado) }\end{array}$} \\
\hline & Estudiantes & Escuelas & $\begin{array}{c}\text { ISE } \\
\text { promedio }\end{array}$ & Estudiantes & Escuelas & $\begin{array}{c}\text { ISE } \\
\text { promedio }\end{array}$ \\
\hline Amazonas & 932 & 23 & 41,46 & 663 & 15 & 43,90 \\
\hline Antioquia & 77708 & 1384 & 50,87 & 56194 & 109 & 53,45 \\
\hline Arauca & 3886 & 83 & 44,90 & 2384 & 56 & 47,08 \\
\hline Atlántico & 31946 & 706 & 50,79 & 26882 & 481 & 52,20 \\
\hline Bogotá & 85619 & 1440 & 57,75 & 80097 & 1060 & 59,36 \\
\hline Bolívar & 31343 & 570 & 46,19 & 23398 & 435 & 48,44 \\
\hline Boyacá & 17502 & 455 & 48,90 & 15093 & 348 & 50,80 \\
\hline Caldas & 11819 & 281 & 49,62 & 8773 & 237 & 51,68 \\
\hline Caquetá & 6629 & 175 & 43,90 & 3976 & 150 & 47,17 \\
\hline Casanare & 7432 & 119 & 48,17 & 522 & 98 & 50,12 \\
\hline Cauca & 2039 & 618 & 42,42 & 1357 & 406 & 45,06 \\
\hline Cesar & 17393 & 349 & 46,89 & 11829 & 225 & 49,08 \\
\hline Chocó & 6109 & 157 & 41,80 & 3748 & 136 & 45,57 \\
\hline Córdoba & 28337 & 548 & 42,72 & 20756 & 408 & 44,37 \\
\hline Cundinamarca & 40023 & 896 & 52,61 & 33332 & 697 & 54,38 \\
\hline Guainía & 672 & 20 & 40,32 & 302 & 16 & 41,57 \\
\hline Guaviare & 1189 & 42 & 42,69 & 895 & 37 & 44,45 \\
\hline Huila & 17792 & 349 & 46,71 & 1218 & 256 & 49,22 \\
\hline La Guajira & 13676 & 230 & 43,15 & 7148 & 136 & 47,28 \\
\hline Magdalena & 22688 & 472 & 45,83 & 15622 & 286 & 47,93 \\
\hline Meta & 1532 & 296 & 49,52 & 10783 & 204 & 51,81 \\
\hline Nariño & 2381 & 1618 & 44,76 & 14969 & 371 & 46,92 \\
\hline Norte de Santander & 20495 & 411 & 48,14 & 15096 & 308 & 51,03 \\
\hline Putumayo & 5107 & 147 & 42,80 & 3436 & 106 & 44,33 \\
\hline Quindío & 6102 & 110 & 52,01 & 5328 & 98 & 54,09 \\
\hline Risaralda & 1101 & 235 & 51,50 & 8861 & 176 & 53,57 \\
\hline San Andrés & 662 & 16 & 52,08 & 592 & 13 & 52,89 \\
\hline Santander & 27692 & 662 & 50,33 & 206 & 478 & 52,42 \\
\hline Sucre & 1506 & 365 & 44,08 & 11039 & 212 & 45,91 \\
\hline Tolima & 20004 & 433 & 47,96 & 15201 & 310 & 50,22 \\
\hline Valle del Cauca & 56285 & 1363 & 52,03 & 43527 & 881 & 53,76 \\
\hline Vaupés & 653 & 15 & 37,11 & 348 & 15 & 38,42 \\
\hline Vichada & 1128 & 42 & 40,39 & 630 & 26 & 42,59 \\
\hline Total/promedio & 646413 & 14630 & 46,68 & 492472 & 9771 & 52,08 \\
\hline
\end{tabular}

Fuente: elaboración propia, a partir de datos de datos de Saber 2016 
La variable criterio utilizada para estimar la segregación escolar es el índice socioeconómico de las familias de los estudiantes. Esta ha sido estimada por el Icfes a partir de un conjunto de variables relativas a las posesiones de las familias, los ingresos y el trabajo del padre y de la madre, y se presenta escalada con una media de 50 puntos y una desviación típica de 10.

Como se ha señalado, para aportar una imagen global de la segregación escolar en Colombia se usarán los cuatro índices más habituales: el índice de disimilitud (Duncan \& Duncan, 1955), el índice de Gorard (Gorard, 2000; 2009; Gorard \& Taylor, 2000; 2002), el índice de raíz cuadrada o de Hutchens (Hutchens, 2001; 2004) y el de aislamiento (Lieberson, 1981). Los tres primeros miden la dimensión de uniformidad y el último, la dimensión de exposición. La razón de esta elección es mostrar las alternativas más habituales para poder comparar estos datos con los de otros estudios similares, pasados o futuros.

Matemáticamente, los cuatro se formulan así:

$$
\begin{aligned}
& \text { Índice de disimilitud } \\
& D=\frac{1}{2} \sum_{i=1}^{k}\left|\frac{x_{1 i}}{X_{1}}-\frac{x_{2 i}}{X_{2}}\right|
\end{aligned}
$$

Índice de aislamiento

$$
A=\sum_{i=1}^{k} \frac{x_{1 i}}{X_{1}} \frac{x_{1 i}}{T_{i}}
$$

\section{Índice de Gorard}

$$
G=\frac{1}{2} \sum_{i=1}^{k}\left|\frac{x_{1 i}}{X_{1}}-\frac{T_{i}}{T}\right|
$$

Índice de raíz cuadrada

$$
H=1-\sum_{i=1}^{k} \sqrt{\frac{x_{2 i}}{X_{2}} \frac{x_{1 i}}{X_{1}}}
$$

Donde:

- $x_{1 i}$ representa el número de estudiantes del grupo minoritario en la escuela $i$

- $x_{2 i}$ es el número de estudiantes del grupo mayoritario en la escuela $i$

- $X_{1}$ y $X_{2}$ son el número total de estudiantes minoritarios y mayoritarios, respectivamente, en todas las escuelas de Colombia (o de los departamentos)

- $T_{i}$, número total de estudiantes en la escuela $i$

- T, número total de estudiantes en Colombia (o en cada uno de los departamentos)

Para dar la imagen más completa posible, se estima la segregación con cuatro grupos minoritarios diferentes: con el $10 \%$ de los estudiantes de familias con menor nivel socioeconómico (P10), el $25 \%$ de los estudiantes de familias con menor nivel socioeconómico (Q1), el $25 \%$ de los estudiantes de familias con mayor nivel socioeconómico (Q4) y, por último, el 10\% de los estudiantes de familias con mayor nivel socioeconómico (P90). 
Pero también se estima el índice de brecha por centiles (o $\mathrm{GCl}$, por sus siglas en inglés: centile gap index) (Watson, 2009), cuya fórmula es:

$$
C G I=1-\frac{4}{N} \sum_{i=1}^{N}\left|p_{i}-p_{\text {medi }}\right|
$$

Donde, para cada estudiante $i, p_{i}$ es el percentil de cada estudiante y $p_{\text {medi }}$ es la mediana de los percentiles de la escuela del estudiante $i$, y $N$ el número de estudiantes total.

Y, por último, el índice de inclusión socioeconómica (IIS) (Murillo, 2016), expresado matemáticamente de la siguiente forma, a partir de un modelo multinivel de dos niveles:

$$
\begin{aligned}
& \operatorname{ISE}_{\mathrm{ij}}=\beta_{0}+\mu_{\mathrm{j}}+\varepsilon_{\mathrm{ij}} \\
& \text { Con: } \\
& {\left[\varepsilon_{\mathrm{ij}}\right] \sim \mathrm{N}\left(0, \Omega_{\varepsilon}\right): \Omega_{\varepsilon}=\left[\sigma_{\varepsilon}^{2}\right]} \\
& {\left[\mu_{\mathrm{j}}\right] \sim \mathrm{N}\left(0, \Omega_{\mu}\right): \Omega_{\mu}=\left[\sigma_{\mu}^{2}\right]}
\end{aligned}
$$

De ahí se estima el coeficiente de correlación intraclase (ICC), que sería el índice de inclusión socioeconómica:

$$
\rho=\frac{\sigma_{\mu}^{2}}{\sigma_{\varepsilon}^{2}+\sigma_{\mu}^{2}}
$$

La razón de usar estos seis índices de forma simultánea es ofrecer una imagen lo más global, completa y fiable posible, de tal manera que estos resultados puedan ser contrastados con estudios previos o posteriores. La incorporación del índice de brecha por centiles y del índice de inclusión socioeconómica es más novedosa y aporta informaciones complementarias. Así, el índice de brecha por centiles usa el índice socioeconómico de las familias para evaluar el promedio de las diferencias percentílicas entre los estudiantes de cada escuela y el percentil mediano en cada una de ellas. El índice de inclusión socioeconómica, por su parte, estima el porcentaje de varianza del índice socioeconómico que es explicado por la variación entre escuelas. En este caso, además, no se necesita dicotomizar la variable criterio, lo que aporta una visión diferente.

Para estimar el perfil de segregación escolar por nivel socioeconómico, así como para analizar la segregación en cada uno de los 32 departamentos, se utiliza solo el índice de Gorard. La razón de esta elección es que este 
índice tiene ventajas claras sobre los otros tres, especialmente la de no estar influido por el tamaño del grupo minoritario (Murillo, 2016).

Para determinar el perfil de segregación se usan 19 grupos minoritarios: el $5 \%$ de los estudiantes de las familias con menos nivel socioeconómico (P5), el 10\% (P10), el 15\% (P15),... hasta el 50\% (P50) y el $45 \%$ de mayor nivel (P55) hasta el $5 \%$ de las de mayor nivel (P95). Así, la estimación de la segregación para una gran cantidad de grupos minoritarios de tamaño creciente no se encuentra en un solo punto (que es la estimación global), sino en una línea de segregación, que es el perfil de segregación escolar por nivel socioeconómico.

Por último, para estimar la segregación en los departamentos se usan los cuatro grupos minoritarios anteriores (P10, Q1, Q4 y P90). Y, en todos los casos, se estima para la educación primaria (básica primaria) y para la educación secundaria inferior (básica secundaria).

\section{Resultados}

Se presentan los resultados organizados en cada uno de los tres objetivos planteados. En primer lugar, una panorámica general de la segregación escolar por nivel socioeconómico en educación primaria y secundaria en Colombia, usando los índices más habituales; en segundo término, los perfiles de segregación escolar, y, por último, el análisis de cada departamento.

\section{Panorámica general de la segregación escolar en Colombia}

El promedio de la segregación escolar por nivel socioeconómico en educación primaria en Colombia es de 0,61 medido con el índice de disimilitud, de 0,51 con el de Gorard, de 0,35 con el de raíz cuadrada, de 0,48 con el de aislamiento, de 0,42 con el de brecha por centiles y de 0,65 con el índice de inclusión socioeconómica (tabla 2). Para la educación secundaria las cifras son levemente superiores: de 0,62 con el de disimilitud, de 0,51 con el de Gorard, 0,36 con el de raíz cuadrada, de 0,48 con el de aislamiento, de 0,42 con el de brecha por centiles y de 0,67 con el índice de inclusión socioeconómica. Con estas cifras es posible hablar de hipersegregación en ambos niveles.

El análisis de la segregación para cada uno de los cuatro grupos minoritarios hace ver, en primer lugar, que la segregación escolar según la dimensión de uniformidad es mayor cuanto más pequeño es el grupo minoritario (fenómeno habitual), y, en segundo término, que es algo más alta para el $25 \%$ y el $10 \%$ de los estudiantes de familias con mayor nivel socioeconómico, en comparación con los grupos análogos de menos nivel. Estos hechos se dan en igual medida en la educación primaria y en la secundaria. 
Tabla 2

Magnitud de la segregación escolar por nivel socioeconómico en Colombia

\begin{tabular}{l|l|l|l|l|l|l|l}
\hline & $\begin{array}{c}\text { Índice de } \\
\text { disimilitud }\end{array}$ & $\begin{array}{c}\text { Índice de } \\
\text { Gorard }\end{array}$ & $\begin{array}{c}\text { Índice de } \\
\text { Hutchens }\end{array}$ & $\begin{array}{c}\text { Índice de } \\
\text { aislamiento }\end{array}$ & $\begin{array}{c}\text { Índice de } \\
\text { brecha por } \\
\text { centiles }\end{array}$ & $\begin{array}{c}\text { índice } \\
\text { de inclusión } \\
\text { socioeconómica }\end{array}$ \\
\hline Primaria & 0,6260 & 0,5633 & 0,3638 & 0,3735 & & \\
\hline P10 & 0,5637 & 0,4227 & 0,2951 & 0,5216 & & \\
\hline Q1 & 0,5870 & 0,4403 & 0,3309 & 0,5530 & & \\
\hline Q4 & 0,6685 & 0,6019 & 0,4236 & 0,4591 & & \\
\hline P90 & $\mathbf{0 , 6 1 1 3}$ & $\mathbf{0 , 5 0 7 1}$ & $\mathbf{0 , 3 5 3 4}$ & $\mathbf{0 , 4 7 6 8}$ & $\mathbf{0 , 4 1 4 9}$ & \\
\hline Promedio & 0,6242 & 0,5618 & 0,3594 & 0,3669 & & \\
\hline Secundaria & 0,5772 & 0,4329 & 0,3028 & 0,5265 & & \\
\hline P10 & 0,5896 & 0,4422 & 0,3313 & 0,5610 & & \\
\hline Q1 & 0,6856 & 0,6171 & 0,4416 & 0,4752 & & \\
\hline Q4 & $\mathbf{0 , 6 1 9 2}$ & $\mathbf{0 , 5 1 3 5}$ & $\mathbf{0 , 3 5 8 8}$ & $\mathbf{0 , 4 8 2 4}$ & $\mathbf{0 , 4 2 1 7}$ & $\mathbf{0 , 6 7 0 5}$ \\
\hline P90 & & & & & \\
\hline Promedio & & & & & \\
\hline
\end{tabular}

Fuente: elaboración propia, a partir de datos de la prueba Saber 2016

\section{Perfiles de segregación escolar en Colombia}

La magnitud de la segregación, sin embargo, no es una cifra aislada. Como se ha señalado, si se estima la segregación para una gran cantidad de grupos minoritarios de tamaño creciente, se encuentra, más que un punto, una línea de segregación: el perfil de segregación escolar por nivel socioeconómico. En este caso, dos, uno para primaria y otro para secundaria. En la figura 1 se han representado ambos perfiles.

Figura 1

Perfiles de segregación escolar por nivel socioeconómico en Colombia.

índice de Gorard para 19 grupos minoritarios

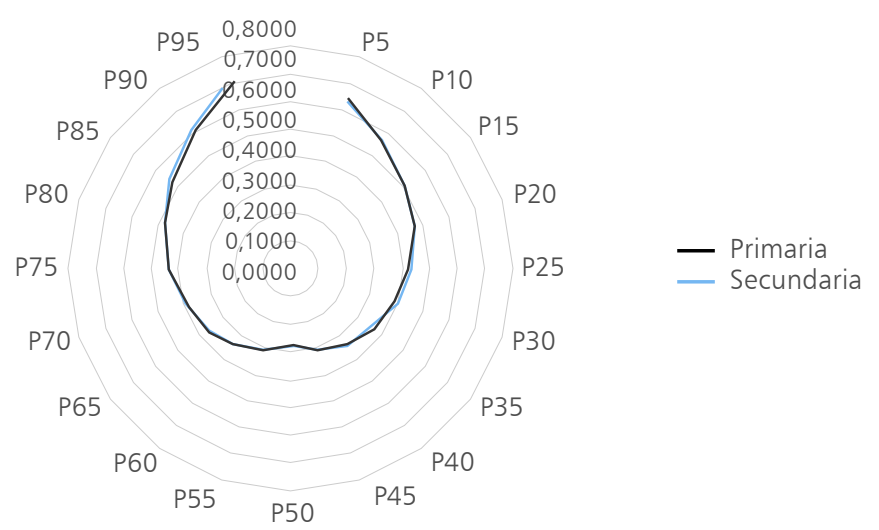


Su análisis confirma con toda claridad dos de las ideas antes formuladas. En primer lugar, se verifica que la segregación escolar es prácticamente idéntica en la educación primaria y en la educación secundaria inferior. Las mínimas diferencias se refieren a que hay mayor segregación en secundaria en los grupos minoritarios de mayor nivel socioeconómico (P90 y 95) y, por el contrario, una mayor segregación escolar en primaria en el P5, el P65 y el P60. En todo caso, las diferencias son muy bajas (tabla 3).

Otra idea que se verifica es que la segregación es más alta para grupos minoritarios de mayor nivel socioeconómico. Precisamente, la figura 1 muestra que la diferencia con la segregación de grupos minoritarios de menos nivel no es tan alta, de tal forma que la imagen es bastante simétrica. La segregación, por tanto, se da en los dos extremos, y en esas líneas hay que tomar las decisiones de política educativa pública.

Tabla 3

Perfiles de segregación escolar por nivel socioeconómico en Colombia

\begin{tabular}{c|c|c}
\hline & Primaria & Secundaria \\
\hline P5 & 0,6391 & 0,6315 \\
\hline P10 & 0,5634 & 0,5618 \\
\hline P15 & 0,5067 & 0,5090 \\
\hline P20 & 0,4606 & 0,4682 \\
\hline P25 & 0,4227 & 0,4329 \\
\hline P30 & 0,3903 & 0,4019 \\
\hline P35 & 0,3627 & 0,3727 \\
\hline P40 & 0,3353 & 0,3434 \\
\hline P45 & 0,3085 & 0,3131 \\
\hline P50 & 0,2817 & 0,2829 \\
\hline P55 & 0,3115 & 0,3101 \\
\hline P60 & 0,3420 & 0,3382 \\
\hline P65 & 0,3728 & 0,3687 \\
\hline P70 & 0,4050 & 0,4028 \\
\hline P75 & 0,4402 & 0,4422 \\
\hline P80 & 0,4818 & 0,4893 \\
\hline P85 & 0,5326 & 0,5456 \\
\hline P90 & 0,6016 & 0,6171 \\
\hline P95 & 0,7053 & 0,7189 \\
\hline Promedio & $\mathbf{0 , 4 4 5 5}$ & $\mathbf{0 , 4 5 0 0}$ \\
\hline & & \\
\hline
\end{tabular}

Fuente: elaboración propia, a partir de datos de la prueba Saber 2016

\section{Segregación escolar en los departamentos colombianos}

El tercer objetivo de esta investigación es estimar la segregación escolar por nivel socioeconómico en cada uno de los 32 departamentos en los que se divide Colombia y Bogotá como distrito capital. Para ello, como se señaló, se utiliza el índice de Gorard con cuatro grupos minoritarios: P10, Q1, Q4 y P90. 
Los resultados apuntan a la existencia de una gran variabilidad en la segregación escolar en función del departamento. Efectivamente, es posible detectar departamentos muy segregados, como Antioquia, Santander o Cundinamarca, con una segregación promedio superior a 0,47 , junto con otros con una segregación realmente baja (por debajo de 0,38), es el caso de San Andrés, Arauca o Casanare (figura 2).

En segundo lugar, se observa con claridad que, en todos los departamentos, la segregación en primaria y en secundaria inferior es parecida, pero no idéntica. En 14 departamentos hay una mayor segregación en primaria que en secundaria, mientras en 18 es mayor en secundaria, por el contrario, en Boyacá estos índices son iguales.

Figura 2

Segregación escolar por nivel socioeconómico en los departamentos de Colombia en educación primaria y en educación secundaria. Índice de Gorard promedio

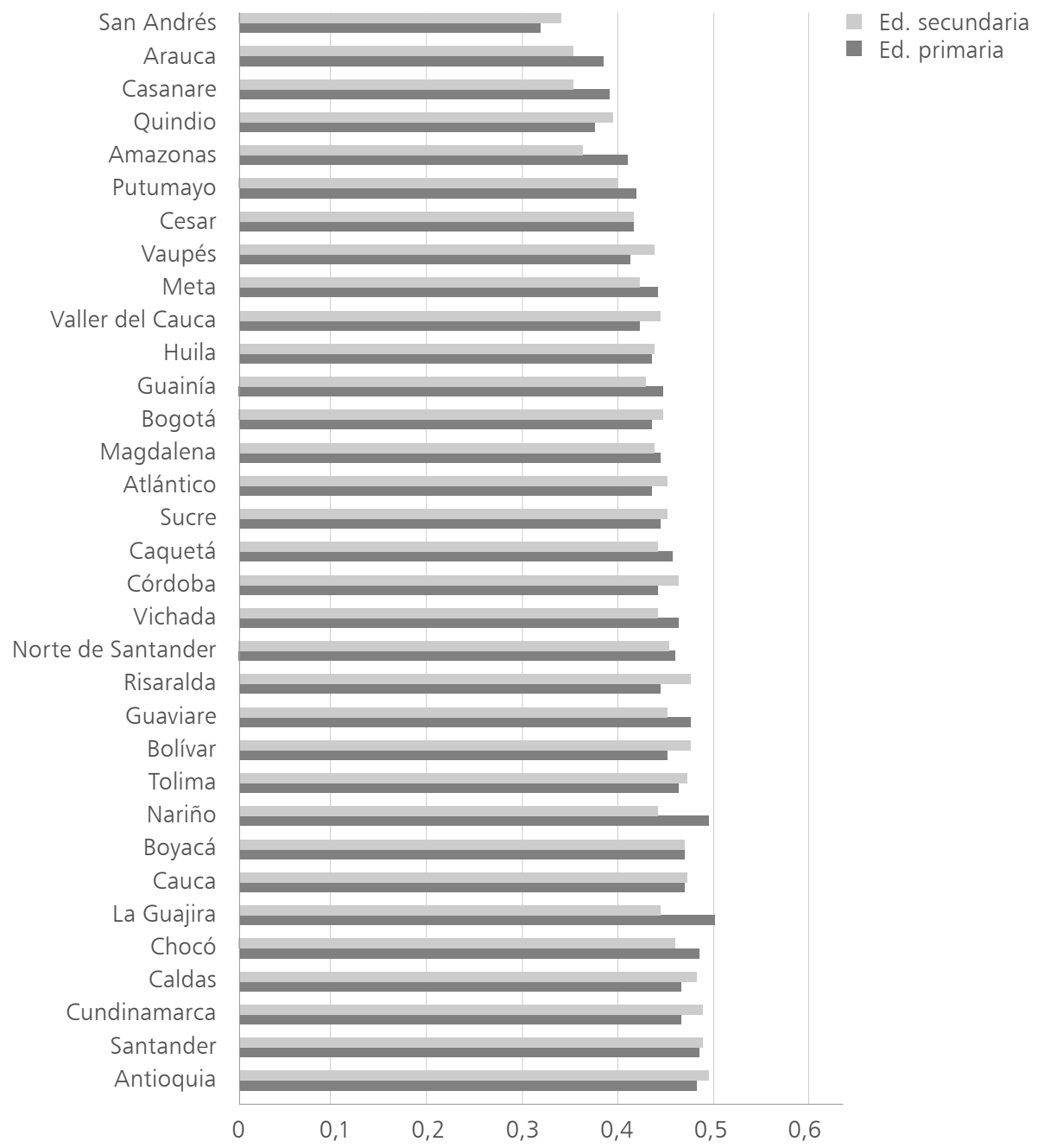

Nota: departamentos ordenados en función de la segregación media de primaria y de secundaria.

Fuente: elaboración propia, a partir de los datos de Saber 2016 
En tercer lugar, al analizar los datos de primaria por grupos minoritarios según los departamentos (tabla 4), se identifica que para el $10 \%$ de los estudiantes con familias de menor nivel socioeconómico, es decir, el percentil 10 (P10), los departamentos con mayor segregación son La Guajira $(0,59)$, Guaviare $(0,58)$ y Nariño $(0,57)$, en comparación con los menos segregados, que son Quindío y San Andrés, con un índice de 0,33 cada uno, y Bogotá, con un índice de 0,37.

Para el 10\% con mayor nivel socioeconómico (P90), los datos permiten tener una visión muy distinta a la revisada anteriormente. Cuando se selecciona a la población de mayor nivel socioeconómico, los departamentos que mayor segregación presentan son Cundinamarca y Bogotá (con un índice de Gorard de 0,62), Caldas y Santander (con una estimación de 0,58); y los departamentos que menos lo hacen son San Andrés $(0,39)$, Casanare y Vaupés (0,43 cada uno).

Este resultado permite tener una visión más amplia de la segregación, pues al tener en cuenta los dos grupos minoritarios: el de mayor y menor nivel socioeconómico, la visión de los departamentos que más segregan es distinta. Tal como ocurre con el distrito capital de Bogotá, que, cuando se selecciona al grupo con menor nivel socioeconómico (P10), es uno de los que menos segrega; pero cuando se toma al 10\% con más recursos, es de los que más segrega.

Ahora, al tomar como referencia al $25 \%$ con mayor y menor nivel socioeconómico (Q1 y Q4), los valores del índice de Gorard varían sustancialmente; donde los resultados para los departamentos más segregados están alrededor de 0,48 y 0,28.

Para el Q1, los departamentos que mayor desproporción evidencian en la distribución de los grupos son nuevamente La Guajira, con una puntuación de 0,48; Guaviare, con un índice de Gorard de 0,46, y Chocó y Caquetá, con un valor de 0,43 cada uno. Los departamentos que menos segregan para la población con menor nivel socioeconómico son San Andrés, Quindío y Arauca, con una estimación del índice de 0,25, 0,27 y 0,29, respectivamente.

Con el fin de obtener las dos caras de la moneda, se estimó el índice para el $25 \%$ con mayor nivel socioeconómico, y se encontraron también resultados muy similares a la elección del P90. En este caso, los departamentos que más segregan por nivel socioeconómico en el Q4 son Cauca y Bogotá, con un resultado de 0,43, y Cundinamarca, Nariño y Caldas, con una estimación del índice de Gorard de 0,42. Los que menos segregan en este grupo son San Andrés $(0,27)$, Casanare $(0,31)$ y Arauca $(0,32)$. 
Tabla 4

Segregación escolar por nivel socioeconómico en $5^{\circ}$ (primaria) en Colombia y sus departamentos. Índice de Gorard para el P10, el Q1, el Q4 y el P10 como grupos minoritarios, y promedio

\begin{tabular}{|c|c|c|c|c|c|}
\hline & P10 & Q1 & Q4 & P90 & Promedio \\
\hline Colombia & 0,5633 & 0,4227 & 0,4403 & 0,6019 & 0,5070 \\
\hline Amazonas & 0,4277 & 0,3015 & 0,3830 & 0,5179 & 0,4075 \\
\hline Antioquia & 0,5375 & 0,4109 & 0,4104 & 0,5715 & 0,4826 \\
\hline Arauca & 0,4549 & 0,2925 & 0,3241 & 0,4590 & 0,3826 \\
\hline Atlántico & 0,4155 & 0,3428 & 0,4128 & 0,5683 & 0,4349 \\
\hline Bogotá & 0,3730 & 0,3126 & 0,4324 & 0,6215 & 0,4349 \\
\hline Bolívar & 0,4724 & 0,3461 & 0,4214 & 0,5678 & 0,4519 \\
\hline Boyacá & 0,5031 & 0,3905 & 0,4114 & 0,5722 & 0,4693 \\
\hline Caldas & 0,4748 & 0,3751 & 0,4261 & 0,5858 & 0,4654 \\
\hline Caquetá & 0,5078 & 0,4309 & 0,3777 & 0,5014 & 0,4545 \\
\hline Casanare & 0,4910 & 0,3236 & 0,3178 & 0,4339 & 0,3916 \\
\hline Cauca & 0,4811 & 0,3862 & 0,4349 & 0,5728 & 0,4687 \\
\hline Cesar & 0,4208 & 0,3141 & 0,3899 & 0,5329 & 0,4144 \\
\hline Choco & 0,5543 & 0,4334 & 0,4068 & 0,5553 & 0,4874 \\
\hline Córdoba & 0,4651 & 0,3628 & 0,3903 & 0,5487 & 0,4418 \\
\hline Cundinamarca & 0,4591 & 0,3493 & 0,4275 & 0,6277 & 0,4659 \\
\hline Guainía & 0,5351 & 0,3735 & 0,4196 & 0,4592 & 0,4469 \\
\hline Guaviare & 0,5812 & 0,4604 & 0,4003 & 0,4596 & 0,4754 \\
\hline Huila & 0,4331 & 0,3453 & 0,4207 & 0,5481 & 0,4368 \\
\hline La Guajira & 0,5981 & 0,4842 & 0,3931 & 0,5253 & 0,5002 \\
\hline Magdalena & 0,4509 & 0,3365 & 0,4113 & 0,5724 & 0,4428 \\
\hline Meta & 0,5089 & 0,3466 & 0,3782 & 0,5338 & 0,4419 \\
\hline Nariño & 0,5709 & 0,4063 & 0,4262 & 0,5757 & 0,4948 \\
\hline Norte de Santander & 0,5293 & 0,3750 & 0,3834 & 0,5511 & 0,4597 \\
\hline Putumayo & 0,4685 & 0,3723 & 0,3594 & 0,4738 & 0,4185 \\
\hline Quindío & 0,3319 & 0,2792 & 0,3512 & 0,5399 & 0,3756 \\
\hline Risaralda & 0,4875 & 0,3794 & 0,3675 & 0,5411 & 0,4439 \\
\hline San Andrés & 0,3320 & 0,2589 & 0,2785 & 0,3950 & 0,3161 \\
\hline Santander & 0,5202 & 0,4135 & 0,4194 & 0,5852 & 0,4846 \\
\hline Sucre & 0,4678 & 0,3543 & 0,3996 & 0,5631 & 0,4462 \\
\hline Tolima & 0,4854 & 0,3736 & 0,4098 & 0,5792 & 0,4620 \\
\hline Valle del Cauca & 0,4313 & 0,3394 & 0,3720 & 0,5486 & 0,4228 \\
\hline Vaupés & 0,4705 & 0,3673 & 0,3641 & 0,4384 & 0,4101 \\
\hline Vichada & 0,5524 & 0,4211 & 0,3972 & 0,4791 & 0,4624 \\
\hline
\end{tabular}

Fuente: elaboración propia, a partir de datos Saber 2016

Para el caso de los estudiantes de secundaria (tabla 5), si se analizan los grupos minoritarios de menor nivel socioeconómico, se identifica que en el P10 los departamentos más segregados son La Guajira (con un índice G de 0,56), Caquetá y Guaviare (con 0,54 cada uno); mientras que Quindío $(0,34)$, Amazonas $(0,35)$ y San Andrés $(0,37)$ son los menos segregados 
en este mismo grupo. En el caso del $25 \%$ de estudiantes con menos nivel socioeconómico (Q1), los departamentos de Santander y Guainía (con un índice de Gorard de 0,42) y Antioquia $(0,41)$ son los más segregados, mientras que Casanare $(0,27)$, San Andrés y Quindío $(0,28$ cada uno) son los menos segregados. Estos dos últimos departamentos se mantienen como los menos segregados en ambos grupos minoritarios (P10 y Q1).

En el caso de los estudiantes con mayor nivel socioeconómico (P90 y Q4), la magnitud del índice de segregación es mayor, como se ha identificado previamente, y los grupos en los extremos se mantienen. Así, los departamentos más segregados son Cundinamarca (índice de Gorard en el P90 de 0,64 y en el Q4 de 0,44), Bogotá (índice en el P90 de 0,63 y en el Q4 de 0,44) y Bolívar (índice en el P90 de 0,60 y en el Q4 de 0,45); mientras que los menos segregados son San Andrés (índice en el P90 de 0,37 y en el Q4 de 0,32), Arauca (índice en el P90 de 0,39 y en el Q4 de 0,30) y Casanare (índice en el P90 de 0,39 y en el Q4 de 0,29).

Tabla 5

Segregación escolar por nivel socioeconómico en $9^{\circ}$ (secundaria) en Colombia y sus departamentos. Índice de Gorard para el P10, el Q1, el Q4 y el P90 como grupos minoritarios, y promedio

\begin{tabular}{|c|c|c|c|c|c|}
\hline & P10 & Q1 & Q4 & P90 & Promedio \\
\hline Colombia & 0,5618 & 0,4329 & 0,4422 & 0,6171 & 0,5136 \\
\hline Amazonas & 0,3505 & 0,2944 & 0,3296 & 0,4703 & 0,3612 \\
\hline Antioquia & 0,5396 & 0,4181 & 0,4174 & 0,5996 & 0,4937 \\
\hline Arauca & 0,4128 & 0,3049 & 0,3008 & 0,3948 & 0,3533 \\
\hline Atlántico & 0,4328 & 0,3653 & 0,4081 & 0,5946 & 0,4502 \\
\hline Bogotá & 0,3818 & 0,3227 & 0,4447 & 0,6314 & 0,4452 \\
\hline Bolívar & 0,4712 & 0,3775 & 0,4501 & 0,6011 & 0,4750 \\
\hline Boyacá & 0,5051 & 0,3831 & 0,4175 & 0,5766 & 0,4706 \\
\hline Caldas & 0,5052 & 0,4099 & 0,4255 & 0,5907 & 0,4828 \\
\hline Caquetá & 0,5490 & 0,3944 & 0,3592 & 0,4704 & 0,4432 \\
\hline Casanare & 0,4398 & 0,2797 & 0,2935 & 0,3966 & 0,3524 \\
\hline Cauca & 0,4833 & 0,3776 & 0,4345 & 0,5953 & 0,4727 \\
\hline Cesar & 0,4315 & 0,3134 & 0,3882 & 0,5245 & 0,4144 \\
\hline Chocó & 0,5182 & 0,3562 & 0,4109 & 0,5556 & 0,4602 \\
\hline Córdoba & 0,4908 & 0,3774 & 0,4066 & 0,5734 & 0,4621 \\
\hline Cundinamarca & 0,4859 & 0,3749 & 0,4425 & 0,6423 & 0,4864 \\
\hline Guainía & 0,4890 & 0,4215 & 0,3697 & 0,4384 & 0,4296 \\
\hline Guaviare & 0,5431 & 0,3623 & 0,3971 & 0,4972 & 0,4499 \\
\hline Huila & 0,4604 & 0,3527 & 0,3999 & 0,5450 & 0,4395 \\
\hline La Guajira & 0,5677 & 0,3612 & 0,3545 & 0,4934 & 0,4442 \\
\hline Magdalena & 0,4399 & 0,3407 & 0,4121 & 0,5655 & 0,4395 \\
\hline Meta & 0,4454 & 0,3287 & 0,3878 & 0,5256 & 0,4219 \\
\hline
\end{tabular}




\begin{tabular}{l|c|c|c|c|c}
\hline & $\mathbf{P 1 0}$ & $\mathbf{Q 1}$ & $\mathbf{Q 4}$ & $\mathbf{P 9 0}$ & Promedio \\
\hline Nariño & 0,4497 & 0,3372 & 0,4086 & 0,5657 & 0,4403 \\
\hline Norte de Santander & 0,4877 & 0,3650 & 0,4073 & 0,5567 & 0,4542 \\
\hline Putumayo & 0,4612 & 0,3533 & 0,3425 & 0,4452 & 0,4006 \\
\hline Quindío & 0,3488 & 0,2885 & 0,3915 & 0,5415 & 0,3926 \\
\hline Risaralda & 0,5172 & 0,3985 & 0,4059 & 0,5809 & 0,4756 \\
\hline San Andrés & 0,3711 & 0,2872 & 0,3277 & 0,3779 & 0,3410 \\
\hline Santander & 0,5386 & 0,4293 & 0,4045 & 0,5773 & 0,4874 \\
\hline Sucre & 0,4881 & 0,3735 & 0,4124 & 0,5309 & 0,4512 \\
\hline Tolima & 0,5057 & 0,4001 & 0,4009 & 0,5811 & 0,4720 \\
\hline Valle del Cauca & 0,4389 & 0,3410 & 0,4031 & 0,5938 & 0,4442 \\
\hline Vaupés & 0,5110 & 0,3678 & 0,3678 & 0,4993 & 0,4365 \\
\hline Vichada & 0,5397 & 0,3864 & 0,3360 & 0,5079 & 0,4425 \\
\hline
\end{tabular}

Fuente: elaboración propia a partir de datos de Saber 2016

De esta forma, la mayor segregación escolar, al menos en su dimensión de uniformidad, se evidencia en los grupos de menor nivel socioeconómico de los departamentos de La Guajira y Guaviare, tanto para primaria como para secundaria; mientras que los departamentos de Bogotá y Cundinamarca son los más segregados para los grupos con mayor nivel socioeconómico en ambos niveles.

\section{Discusión y conclusiones}

En coherencia con otros trabajos, esta investigación ha encontrado que Colombia tiene una segregación escolar por nivel socioeconómico que puede ser considerada como muy alta (Massey \& Denton, 1988) - hipersegregación en terminología de Glaeser \& Vigdor (2001) —, tanto en educación primaria como en educación secundaria, con cierta tendencia a ser superior para estudiantes de familias con mayor nivel socioeconómico.

Para educación primaria, la segregación escolar promedio encontrada en este estudio es de 0,61, según el índice D; de 0,51, según el índice G; de 0,35, según el índice $H$; de 0,48, según el índice $A$ y, por último, de 0,65, según el índice de inclusión socioeconómica. Estas cifras son coherentes con las aportadas por Murillo (2016) con los datos del Terce, aunque algo más altas. El investigador encontró que la segregación escolar para el conjunto de Colombia es de 0,59, 0,44, 0,35, 0,54 y 0,57, según los índices de disimilitud, de Gorard, de raíz cuadrada, de aislamiento y de inclusión socioeconómica, respectivamente. Murillo \& Martínez-Garrido (2017), por su parte, encontraron que la segregación en Colombia, con el índice de disimilitud es de 0,59 y que el índice de disimilitud en el Q1 es de 0,53 y en el Q4 de 0,66; mientras que en este estudio el Q1 es de 0,56 y en el Q4 es de 0,59. 
Para la educación secundaria, es posible comparar los resultados de esta investigación con la obtenida por diversos trabajos que han usado el PISA. Así, Murillo et al. (2018) encuentran que la segregación escolar en Colombia (usando el índice de Gorard) es de 0,47, para el P10; de 0,35, para el Q1; de 0,38, para el Q4, y de 0,53, para el P90. Estas cifras son algo más bajas que las encontradas en este estudio: $0,56,0,43,0,44$ y 0,62, respectivamente. Comparaciones análogas pueden realizarse con los datos correspondientes al PISA 2015 de Vazquez (2016) y Krüger (2019).

Las diferencias encontradas tanto en los trabajos en educación primaria (usando la base de datos del Terce) y los de educación secundaria (con datos del PISA) pueden deberse a varias razones. En primer lugar, a la variable de criterio utilizada: este estudio trabaja con el índice socioeconómico estimado por el Icfes, mientras que Murillo (2016) y Murillo \& Martínez-Garrido (2017) usan el índice socioeconómico y cultural (ISECF), calculado por el Laboratorio Latinoamericano de Evaluación de la Calidad de la Educación (Llece). Krüger (2019), Murillo et al. (2018) y Vazquez (2016), en educación secundaria, usan el ESCS (economic, social and cultural status), estimado por el PISA. También es conveniente recordar que en los análisis derivados del Terce, la muestra es de 8188 estudiantes y en los del PISA 2015 es de 11795 estudiantes; mientras que en este trabajo se usan datos censales con lo que se analizan 646413 estudiantes de primaria y 492472 de secundaria. Además, los datos de este trabajo corresponden al año 2016, mientras que el Terce recogió los datos en el 2013 y el PISA en el 2015.

Este estudio, además, ha realizado dos aproximaciones a la segregación escolar por nivel socioeconómico en Colombia inéditas hasta este momento. Por un lado, se estimó el perfil de la segregación escolar en Colombia, y se encontró que la segregación para los estudiantes con familias de menor nivel socioeconómico es casi igual que para los de familias con más recursos. Esos datos son muy diferentes a los estimados en otros contextos, como España (Murillo \& Martínez-Garrido, 2019) o Uruguay (Murillo \& Graña, 2020), donde la segregación es mayor para los estudiantes de mayores recursos. Por esto, se piensa que el gran problema es la segregación de los estudiantes más desfavorecidos.

El otro calculo novedoso para Colombia es la estimación de la magnitud de la segregación para cada uno de los departamentos colombianos, tanto en educación primaria como en secundaria, y para varios grupos minoritarios. Así, por ejemplo, se encontró que el distrito capital de Bogotá, comparado con los otros departamentos, tiene la menor segregación para los estudiantes con familias de menor nivel socioeconómico (P10), tanto para primaria como para secundaria; sin embargo, es uno de los más 
segregados en los grupos de mayor nivel socioeconómico (Q4 y P90) en ambos niveles. En este último grupo, lo acompaña Cundinamarca, como uno de los más segregados en los grupos de mayor nivel socioeconómico. En contraposición, San Andrés es el departamento menos segregado, en ambos niveles y en todos los grupos analizados.

Con todo ello, es posible establecer una radiografía de la segregación escolar en Colombia basada en tres elementos:

- Colombia tiene unos índices de segregación escolar muy altos, de los más altos de América Latina y, con ello, quizá del mundo, tanto en educación primaria como en secundaria.

- Aunque la segregación es alta tanto para estudiantes de familias con más recursos como para estudiantes de familias con menos, su principal desafío es la segregación escolar de los estudiantes de los grupos más desfavorecidos.

- Existe una gran variabilidad en la segregación de un departamento a otro, pues cada uno tiene características diferentes.

La presente investigación tiene como mayor virtud el haber podido estimar la segregación escolar en Colombia a partir de datos censales, tanto para educación primaria como para secundaria. Ese hecho ha permitido estimar tanto el perfil de segregación como profundizar en la segregación por departamentos. Sin embargo, la preocupante situación encontrada deja la necesidad de conocer más. Así, es necesario obtener mayores evidencias empíricas sobre la segregación escolar en Colombia: identificar causas y estudiar sus consecuencias, tanto académicas como sociales, a corto, medio y largo plazo. Pero también es necesario profundizar en temas tales como la segregación escolar en poblaciones indígenas, estudiantes afrodescendientes y grupos desplazados, inmigrantes y/o refugiados. Si bien se trata de grupos minoritarios dentro del sistema educativo, su vulnerabilidad demanda visibilizar la magnitud de su segregación y cómo esto afecta a la igualdad de oportunidades y la calidad educativa.

Las muchas cifras aportadas en esta investigación llevan a una sola idea conclusiva: Colombia tiene un grave problema de segregación escolar, situándose entre los países con más segregación de América Latina y, con ello, del planeta. Desde una mirada a los planes educativos nacionales y locales de Colombia de los últimos años se evidencia que la reducción de la segregación escolar no ha sido una prioridad (Organisation for Economic Co-operation and Development, 2016), y si este problema no se visibiliza, se hace cada vez más grave (García-Villegas et al., 2013). De poco servirá la preocupación por mejorar la calidad de la educación si esta sigue lastrada 
por graves problemas de inequidad. Es necesario que Colombia comience a situar a la segregación escolar como uno de los desafíos educativos más importantes a abordar en los próximos años. Por lo mismo, creemos que una vez identificado - y denunciado - el problema, es prioritario que se empiecen a tomar las decisiones de política que afronten esta situación de manera contextualizada en cada uno de sus departamentos y en ambos niveles educativos, y que además se prioricen, entre otras medidas, a las escuelas menos favorecidas, las cuales tienen la mayor necesidad de contar con más y mejores recursos, así como con profesores bien capacitados y motivados (Organisation for Economic Co-operation and Development, 2016). Un sistema educativo segregado debilita la posibilidad de garantizar la igualdad de oportunidades para todos sus estudiantes y a la vez hace muy difícil construir, en el mediano y largo plazo, sociedades más justas e inclusivas.

\section{Sobre los autores}

F. Javier Murillo es director de la Cátedra Unesco en Educación para la Justicia Social de la Universidad Autónoma de Madrid, profesor de Métodos de Investigación en Educación en la UAM, coordinador del grupo de investigación Cambio Educativo para la Justicia Social y de la Red Iberoamericana de Investigación sobre Cambio y Eficacia Escolar (Rinace).

Sandra Carrillo-Luna es investigadora en temas relacionados con las políticas educativas sobre segregación escolar, ciudadanía, género y desigualdades sociales; miembro de la Sociedad de Investigación de la Educación Peruana (SIEP); docente contratada del Departamento de Psicología de la PUCP, y coordinadora de programas y proyectos educativos en la Oficina de la Unesco Lima.

\section{Referencias}

Agirdag, O., Van Avermaet, P. \& Van Houtte, M. (2013). School segregation and math achievement: A mixed-method study on the role of self-fulfilling prophecies. Teachers College Record, 115(3), 1-50. https://citeseerx.ist.psu.edu/ viewdoc/download?doi=10.1.1.706.5666\&rep $=$ rep1\&type $=p d f$

Departamento Administrativo Nacional de Estadística (DANE). (2016). Gran Encuesta Integrada de Hogares (GEIH) 2016. Autor.

Departamento Administrativo Nacional de Estadística (DANE). (2019). Bases de datos de educación formal años 2019. Autor.

Duarte, J., Bos, M. S. \& Moreno, J. M. (2012). Calidad, igualdad y equidad en la educación colombiana. BID.

Duncan, O. B. \& Duncan, B. (1955). A methodological analysis of segregation indexes. American Sociological Review, 20(2), 210-217. https://doi. org/10.2307/2088328 
Dupriez, V. \& Dumay, X. (2006). Inequalities in school systems: Effect of school structure or of society structure? Comparative Education, 42(2), 243-260. https://doi.org/10.1080/03050060600628074

García-Villegas, M., Espinosa-Restrepo, J. R., Jiménez-Ángel, F. \& Parra-Heredia, J. D. (2013). Separados y desiguales. Educación y clases sociales en Colombia. Centro de Estudios de Derecho, Justicia y Sociedad (Dejusticia).

Glaeser, E. L. y Vigdor, J. L. (2001). Racial segregation: Promising news. En B. Katz \& R. E. Lang (Eds.), Redefining Urban \& Suburban America: Evidence from Census (pp. 211-234). Brookings Institution.

Gorard, S. (2000). Education and Social Justice. University of Wales Press.

Gorard, S. (2009). Does the index of segregation matter? The composition of secondary schools in England since 1996. British Educational Research Journal, 35(4), 639-652. https://doi.org/10.1080/01411920802642389

Gorard, S. (2014). The link between academies in England, pupil outcomes and local patterns of socio-economic segregation between schools. Research Papers in Education, 29(3), 268-284. https://doi.org/10.1080/02671522.2 014.885726

Gorard, S. \& Taylor, C. (2000). A comparison of segregation indices used for assessing the socio-economic composition of schools. Cardiff UniversitySchool of Social Sciences / Economic and Social Research Council.

Gorard, S. \& Taylor, C. (2002). What is segregation? A comparison of measures in terms of "strong" and "weak" compositional invariance. Sociology, 36(4), 875-895. https://doi.org/10.1177/003803850203600405

Granvik-Saminathen, M., Brolin-Låftman, S., Almquist, Y. B. \& Modin, B. (2018). Effective schools, school segregation, and the link with school achievement. School Effectiveness and School Improvement, 29(3), 464-484. https://doi. org/10.1080/09243453.2018.1470988

Hutchens, R. (2001). Numerical measures of segregation: Desirable properties and their implications. Mathematical Social Sciences, 42(1), 13-29. https:// doi.org/10.1016/s0165-4896(00)00070-6

Hutchens, R. (2004). One measure of segregation. International Economic Review, 45(2), 555-578. https://doi.org/10.1111/j.1468-2354.2004.00136.x

Krüger, N. (2019). La segregación por nivel socioeconómico como dimensión de la exclusión educativa: 15 años de evolución en América Latina. Archivos Analíticos de Políticas Educativas, 27(8), 1-37. https://doi.org/10.14507/ epaa.27.3577

Lieberson, S. (1981). An asymmetrical approach to segregation. En C. Peach (Ed.), Ethnic segregation in cities (pp. 61-83). Croom-Helm.

Massey, D. S. \& Denton, N. A. (1988). The dimensions of residential segregation. Social Forces, 67(2), 281-315. https://doi.org/10.2307/2579183

Mickelson, R. (2018). A synthesis of social science research on the effects of ethnic, racial and socioeconomic composition of schools in the United States. En X. Bonal \& C. Bellei (Eds.), Understanding School Segregation: Patterns, Causes and Consequences of Spatial Inequalities in Education (pp. 123-154). Bloomsbury.

Murillo, F. J. (2016). Midiendo la segregación escolar en América Latina. Un análisis metodológico utilizando el TERCE. REICE. Revista Iberoamericana 
sobre Calidad, Eficacia y Cambio en Educación, 14(4), 33-60. https://doi. org/10.15366/reice2016.14.4.002

Murillo, F. J. \& Graña, R. (2020). Una panorámica de la segregación escolar por nivel socioeconómico en Uruguay. Cuadernos de Investigación Educativa, 11(1), 15-35. https://doi.org/10.18861/cied.2020.11.1.2941

Murillo, F. J. \& Martínez-Garrido, C. (2017). Estimación de la magnitud de la segregación escolar en América Latina. magis. Revista Internacional de Investigación Educativa, 9(19), 11-30. https://doi.org/10.11144/javeriana.m9-19.emse

Murillo, F. J. \& Martínez-Garrido, C. (2019). Perfiles de segregación escolar por nivel socioeconómico en España y sus comunidades autónomas. Relieve, 25(1). https://doi.org/10.7203/relieve.25.1.12917

Murillo, F. J., Duk, C. \& Martínez-Garrido, C. (2018). Evolución de la segregación socioeconómica de las escuelas de América Latina. Estudios Pedagógicos, 44(1), 157-179. https://doi.org/10.4067/S0718-07052018000100157

Organisation for Economic Co-operation and Development (OECD). (2012). Higher Education in Regional and City Development: Antioquia, Colombia. OECD. https://doi.org/10.1787/9789264179028-en

Organisation for Economic Co-operation and Development (OECD). (2016). Education in Colombia. Reviews of National Policies for Education. OECD. https://doi.org/10.1787/9789264250604-en

Organisation for Economic Co-operation and Development (OECD). (2019). PISA 2018. Insights and Interpretations. OECD. https://www.oecd.org/pisa/ PISA\%202018\%20Insights\%20and\%20Interpretations\%20FINAL\%20PDF.pdf

Palardy, G. J. (2013). High school socioeconomic segregation and student attainment. American Educational Research Journal, 50(4), 714-754. https://doi. org/10.3102/0002831213481240

Radinger, T., Echazarra, A., Guerrero, G. \& Valenzuela, J. P. (2018). OECD Reviews of School Resources: Colombia 2018. OECD. https://doi.org/10.1787/ 9789264303751-en

Valenzuela, J. P., Bellei, C. \& Ríos, D. D. L. (2014). Socioeconomic school segregation in a market-oriented educational system. The case of Chile. Journal of Education Policy, 29(2), 217-241. https://doi.org/10.1080/02680939.20 13.806995

Vazquez, E. (2016). Segregación escolar por nivel socioeconómico. Midiendo el fenómeno y explorando sus determinantes. Económica, 62, 121-184. https://econpapers.repec.org/article/lapjournl/604.htm

Watson, T. (2009). Inequality and the measurement of residential segregation by income in American neighborhoods. Review of Income and Wealth, 55(3), 820-844. https://doi.org/10.1111/j.1475-4991.2009.00346.x 